\title{
Schur-geometric convexity of the generalized Gini-Heronian means involving three parameters
}

\author{
Yong-Ping Deng ${ }^{1}$, Yu-Ming Chu ${ }^{2 *}$, Shan-He Wu' ${ }^{1}$ Lokenath Debnath ${ }^{3}$ and Deng $\mathrm{He}^{4}$
}

\section{"Correspondence:}

chuyuming2005@126.com

${ }^{2} \mathrm{School}$ of Mathematics and

Computation Science, Hunan City

University, Yiyang, 413000, P.R. China

Full list of author information is

available at the end of the article

\begin{abstract}
In this paper, we give a unified generalization of the Gini means and Heronian means. The Schur-geometric convexity of the generalized Gini-Heronian means are investigated. Our result generalizes an earlier result given by Shi et al. (J. Inequal. Appl. 2008:879273, 2008). At the end of the paper, two new inequalities related to the generalized Gini-Heronian means are established to illustrate the applicability of the given result.
\end{abstract}

MSC: 26D15; 26E60; 26A51

Keywords: generalized Gini-Heronian means; Schur-geometric convex; Schur-geometric concave; parameter; inequality

\section{Introduction}

The Schur convexity of functions relating to special means have been investigated by many mathematicians, a number of results can be found in the monograph of Marshall and Olkin [1]. As a supplement to the Schur convexity of functions, the Schur-geometric convexity of functions was recently studied by Shi and Zhang [2-4], Zhang and Yang [5] and Chu et al. [6], some related results have been found to have an important application in discovering and proving the inequalities for special means. The purpose of this paper is to investigate the Schur-geometric convexity of functions related to Gini means and Heronian means. Besides, as application, we establish two new inequalities for generalized Gini-Heronian means. Our result generalizes an earlier result given by Shi et al. in [7].

In what follows, we denote the set of real numbers by $\mathbb{R}$, the set of nonnegative real numbers by $\mathbb{R}_{+}$, the set of positive real numbers by $\mathbb{R}_{++}$, and the set of nonpositive real numbers by $\mathbb{R}_{-}$.

Let $(r, s) \in \mathbb{R}^{2},(x, y) \in \mathbb{R}_{++}^{2}$; the classical Gini means are defined by (see [8])

$$
G(r, s ; x, y)= \begin{cases}\left(\frac{x^{r}+y^{r}}{x^{s}+y^{s}}\right)^{1 /(r-s)}, & r \neq s, \\ \exp \left(\frac{x^{r} \ln x+y^{r} \ln y}{x^{r}+y^{r}}\right), & r=s .\end{cases}
$$

In 2007, Sándor [9] investigated the Schur convexity of $G(r, s ; x, y)$ with respect to $(r, s)$, and obtained the following result.

Theorem A For fixed $(x, y) \in \mathbb{R}_{++}^{2}$ and $x \neq y$, the Gini means $G(r, s ; x, y)$ is Schur concave with respect to $(r, s)$ on $\mathbb{R}_{+}^{2}$, and $G(r, s ; x, y)$ is Schur convex with respect to $(r, s)$ on $\mathbb{R}_{-}^{2}$.

O2014 Deng et al.; licensee Springer. This is an Open Access article distributed under the terms of the Creative Commons Attribution License (http://creativecommons.org/licenses/by/2.0), which permits unrestricted use, distribution, and reproduction in any medium, provided the original work is properly cited. 
In the same year, Wang [10] proved the Schur convexity and the Schur-geometric convexity of $G(r, s ; x, y)$ with respect to $(x, y)$ on $\mathbb{R}_{++}^{2}$, as follows.

Theorem B The Gini means $G(r, s ; x, y)$ is Schur convex with respect to $(x, y)$ on $\mathbb{R}_{++}^{2}$ if and only if $(r, s) \in\{(r, s) \mid r \geq 0, s \geq 0, r+s \geq 1\}$, and $G(r, s ; x, y)$ is Schur concave with respect to $(x, y)$ on $\mathbb{R}_{++}^{2}$ if and only if $(r, s) \in\{(r, s) \mid r \leq 0, r+s \leq 1\} \cup\{(r, s) \mid s \leq 0, r+s \leq 1\}$.

Theorem C The Gini means $G(r, s ; x, y)$ is Schur-geometric convex with respect to $(x, y)$ on $\mathbb{R}_{++}^{2}$ if and only if $(r, s) \in\{(r, s) \mid r+s \geq 0\}$, and $G(r, s ; x, y)$ is Schur-geometric concave with respect to $(x, y)$ on $\mathbb{R}_{++}^{2}$ if and only if $(r, s) \in\{(r, s) \mid r+s \leq 0\}$.

Some different proofs concerning the Schur convexity of $G(r, s ; x, y)$ were given by Shi et al. [11], Chu and Xia [12], respectively.

Xia and Chu [13] presented the necessary and sufficient condition for the Schurharmonic-convexity of $G(r, s ; x, y)$ with respect to $(x, y)$ on $\mathbb{R}_{++}^{2}$.

A further discussion on the Schur-power-convexity of $G(r, s ; x, y)$ with respect to $(x, y)$ on $\mathbb{R}_{++}^{2}$ was given by Yang [14]. Meanwhile, the necessary and sufficient condition for the Schur-power-convexity of $G(r, s ; x, y)$ was obtained.

Let $(x, y) \in \mathbb{R}_{++}^{2}$; the classical Heronian means is defined by (see [15])

$$
H_{e}(x, y)=\frac{x+\sqrt{x y}+y}{3} .
$$

In 1999, Mao [16] gave the definition of the dual Heronian means as follows:

$$
\widetilde{H}_{e}(x, y)=\frac{x+4 \sqrt{x y}+y}{6} .
$$

In 2001, Janous [17] considered a unified generalization of the Heronian means $H_{e}(x, y)$ and dual Heronian means $\widetilde{H}_{e}(x, y)$, and presented the following Heronian-type means with a parameter $w$ :

$$
H_{w}(x, y)= \begin{cases}\frac{x+w \sqrt{x y}+y}{w+2}, & 0 \leq w<\infty \\ \sqrt{x y}, & w=\infty\end{cases}
$$

Jia and Cao [18] investigated the exponential generalization of the Heronian means,

$$
H_{p}(x, y)= \begin{cases}\left(\frac{x^{p}+(x y)^{p / 2}+y^{p}}{3}\right)^{1 / p}, & p \neq 0, \\ \sqrt{x y}, & p=0,\end{cases}
$$

and established some related inequalities. Moreover, the monotonicity and Schur convexity of the Heronian means $H_{p}(x, y)$ were discussed by Li et al. in [19].

Shi et al. [7] discussed the Schur convexity and Schur-geometric convexity of a further generalization of the Heronian means given by

$$
H_{p, w}(x, y)= \begin{cases}\left(\frac{x^{p}+w(x y)^{p / 2}+y^{p}}{w+2}\right)^{1 / p}, & p \neq 0, \\ \sqrt{x y}, & p=0,\end{cases}
$$

and obtained some significant results, asserted by Theorems $\mathrm{D}$ and $\mathrm{E}$ below. 
Theorem D For fixed $(p, w) \in \mathbb{R}^{2}$,

(1) if $(p, w) \in\{(p, w) \mid p \geq 2,0 \leq w \leq 2\}$, then $H_{p, w}(x, y)$ is Schur convex for $(x, y) \in \mathbb{R}_{+}^{2}$;

(2) if $(p, w) \in\{(p, w) \mid p \leq 1, w \geq 0\} \cup\{(p, w) \mid 1<p \leq 3 / 2, w \geq 1\} \cup\{(p, w) \mid 3 / 2<p \leq 2$, $w \geq 2\}$, then $H_{p, w}(x, y)$ is Schur concave for $(x, y) \in \mathbb{R}_{+}^{2}$.

Theorem E For fixed $(p, w) \in \mathbb{R}^{2}$,

(1) if $(p, w) \in\{(p, w) \mid p>0, w \geq 0\}$, then $H_{p, w}(x, y)$ is Schur-geometric convex for $(x, y) \in \mathbb{R}_{++}^{2}$

(2) if $(p, w) \in\{(p, w) \mid p<0, w \geq 0\}$, then $H_{p, w}(x, y)$ is Schur-geometric concave for $(x, y) \in \mathbb{R}_{++}^{2}$.

As a further investigation of Theorem D, Fu et al. [20] gave the necessary and sufficient condition for the Schur convexity of the generalized Heronian means $H_{p, w}(x, y)$. Yang [21] investigated the Schur-power-convexity of $H_{p, w}(x, y)$ with respect to $(x, y) \in \mathbb{R}_{++}^{2}$. Mortici [22] studied certain special means relating to convex functions.

In this paper, we shall generalize the Gini means $G(r, s ; x, y)$ and the Heronian means $H_{p, w}(x, y)$ in a unified form. For this purpose we define a generalized Gini-Heronian means containing three parameters $p, q$, and $w$, as follows:

$$
H_{p, q, w}(x, y)= \begin{cases}\left(\frac{\left.x^{p}+w(x y)\right)^{p / 2}+y^{p}}{\left.x^{q}+w(x)\right)^{q / 2}+y^{q}}\right)^{1 /(p-q),} & p \neq q, \\ \exp \left(\frac{x^{p} \ln x+(w / 2)(x y)^{p / 2} \ln (x y)+y^{p} \ln y}{x^{p}+w(x y)^{p / 2}+y^{p}}\right), & p=q,\end{cases}
$$

where $(p, q) \in \mathbb{R}^{2},(x, y) \in \mathbb{R}_{++}^{2}$.

The Schur-geometric convexity of the generalized Gini-Heronian means will be discussed in Section 3. As applications, several inequalities related to generalized GiniHeronian means are established in Section 4.

\section{Definitions and lemmas}

We introduce and establish several definitions and lemmas, which will be used in the proofs of the main results in Sections 3 and 4.

Definition 1 (see [1]) For any $x=\left(x_{1}, x_{2}, \ldots, x_{n}\right), y=\left(y_{1}, y_{2}, \ldots, y_{n}\right) \in \mathbb{R}^{n}$, let $x_{[1]} \geq x_{[2]} \geq$ $\cdots \geq x_{[n]}$ and $y_{[1]} \geq y_{[2]} \geq \cdots \geq y_{[n]}$ denote the components of $x$ and $y$ in decreasing order, respectively.

The $n$-tuple $y$ is said to majorize $x$ (or $x$ is to be majorized by $y$ ), in symbols $x \prec y$, if

$$
\sum_{i=1}^{k} x_{[i]} \leq \sum_{i=1}^{k} y_{[i]} \text { holds } \text { for } k=1,2, \ldots, n-1 \quad \text { and } \quad \sum_{i=1}^{n} x_{i}=\sum_{i=1}^{n} y_{i} .
$$

Definition 2 (see [23]) For any $x=\left(x_{1}, x_{2}, \ldots, x_{n}\right), y=\left(y_{1}, y_{2}, \ldots, y_{n}\right) \in \Omega\left(\Omega \subset \mathbb{R}_{++}^{n}\right), \phi$ : $\Omega \rightarrow \mathbb{R}$ is said to be a Schur-geometric convex function on $\Omega$ if $\left(\ln x_{1}, \ln x_{2}, \ldots, \ln x_{n}\right) \prec$ $\left(\ln y_{1}, \ln y_{2}, \ldots, \ln y_{n}\right)$ on $\Omega$ implies $\phi(x) \leq \phi(y), \phi$ is said to be a Schur-geometric concave function on $\Omega$ if and only if $-\phi$ is a Schur-geometric convex function.

Definition 3 (see [23]) For any $x=\left(x_{1}, x_{2}, \ldots, x_{n}\right), y=\left(y_{1}, y_{2}, \ldots, y_{n}\right) \in \Omega\left(\Omega \subset \mathbb{R}_{++}^{n}\right), \Omega$ is said to be a geometrically convex set if $\left(x_{1}^{\alpha} y_{1}^{\beta}, x_{2}^{\alpha} y_{2}^{\beta}, \ldots, x_{n}^{\alpha} y_{n}^{\beta}\right) \in \Omega$ for all $x, y \in \Omega, \alpha, \beta \in[0,1]$ with $\alpha+\beta=1$. 
Lemma 1 (see [23]) Let $\Omega\left(\Omega \subset \mathbb{R}_{++}^{n}\right)$ be symmetric and have a nonempty interior set $\Omega^{\circ}$, and let $\phi: \Omega \rightarrow \mathbb{R}$ be continuous on $\Omega$ and differentiable in $\Omega^{\circ}$. If $\phi$ is symmetric on $\Omega$ and

$$
\left(x_{1}-x_{2}\right)\left(x_{1} \frac{\partial \phi}{\partial x_{1}}-x_{2} \frac{\partial \phi}{\partial x_{2}}\right) \geq 0(\leq 0)
$$

holds for any $\left(x_{1}, x_{2}, \ldots, x_{n}\right) \in \Omega^{o}$, then $\phi$ is a Schur-geometric convex (Schur-geometric concave) function.

Lemma 2 (see [7]) Let $a \leq b, u(t)=t b+(1-t) a, v(t)=t a+(1-t) b, 1 / 2 \leq t_{2} \leq t_{1} \leq 1$ or $0 \leq t_{1} \leq t_{2} \leq 1 / 2$, then

$$
\left(\frac{a+b}{2}, \frac{a+b}{2}\right) \prec\left(u\left(t_{2}\right), v\left(t_{2}\right)\right) \prec\left(u\left(t_{1}\right), v\left(t_{1}\right)\right) \prec(a, b) .
$$

Lemma 3 Let $p, q \in \mathbb{R}, p>q, \lambda \geq 1$, and let

$$
g_{1}(\lambda)=p \lambda^{p}-q \lambda^{p / 2+q / 2}+q \lambda^{p / 2-q / 2}-p .
$$

Then $g_{1}(\lambda) \geq 0$ for $p+q \geq 0$, and $g_{1}(\lambda) \leq 0$ for $p+q \leq 0$.

Proof Let $f_{1}(\lambda)=2 \lambda^{1-(p / 2-q / 2)} g_{1}^{\prime}(\lambda), f_{2}(\lambda)=\lambda^{1-q} f_{1}^{\prime}(\lambda)$. Straightforward computation yields

$$
\begin{aligned}
& f_{1}(\lambda)=2 p^{2} \lambda^{p / 2+q / 2}-q(p+q) \lambda^{q}+q(p-q), \\
& f_{1}(1)=2(p-q)(p+q), \\
& f_{2}(\lambda)=\lambda^{1-q} f_{1}^{\prime}(\lambda)=p^{2}(p+q) \lambda^{p / 2-q / 2}-q^{2}(p+q), \\
& f_{2}(1)=(p-q)(p+q)^{2}, \\
& f_{2}^{\prime}(\lambda)=\frac{1}{2} p^{2}(p+q)(p-q) \lambda^{p / 2-q / 2-1} .
\end{aligned}
$$

Case 1. $p q \neq 0$.

(1) If $p+q=0$, then

$$
g_{1}(\lambda)=p \lambda^{p}+p \lambda^{p / 2-p / 2}-p \lambda^{p / 2+p / 2}-p=0 .
$$

(2) If $p+q>0$, then from the expressions $f_{2}^{\prime}(\lambda), f_{2}(1), f_{1}(1)$ above we find that, for $\lambda \geq 1$,

$$
f_{2}^{\prime}(\lambda)>0, \quad f_{2}(1)>0, \quad f_{1}(1)>0 .
$$

We thus conclude that the functions $f_{2}(\lambda), f_{1}(\lambda)$, and $g_{1}(\lambda)$ are increasing for $\lambda \in[1,+\infty)$. In fact, for $\lambda \geq 1$, one has

$$
\begin{aligned}
f_{2}^{\prime}(\lambda)>0 & \Longrightarrow f_{2}(\lambda)>0 \quad \Longrightarrow \quad f_{1}^{\prime}(\lambda)>0 \quad \Longrightarrow \quad f_{1}(\lambda)>0 \\
& \Longrightarrow \quad g_{1}^{\prime}(\lambda)>0 \quad \Longrightarrow \quad g_{1}(\lambda) \geq g_{1}(1)=0 .
\end{aligned}
$$

(3) If $p+q<0$, then

$$
f_{2}^{\prime}(\lambda)<0, \quad f_{2}(1)>0, \quad \lim _{\lambda \rightarrow+\infty} \frac{f_{2}(\lambda)}{\lambda^{p / 2-q / 2}}=p^{2}(p+q)<0 .
$$


According to the above relations and the continuity of $f_{2}(\lambda)$, we deduce that there exists $\lambda_{1} \in(1,+\infty)$ such that $f_{2}\left(\lambda_{1}\right)=0$, which leads us to $f_{2}(\lambda)>0$ for $\lambda \in\left[1, \lambda_{1}\right)$, and $f_{2}(\lambda)<0$ for $\lambda \in\left(\lambda_{1},+\infty\right)$.

Thus, we deduce that $f_{1}(\lambda)$ is increasing on $\left[1, \lambda_{1}\right)$ and decreasing on $\left(\lambda_{1},+\infty\right)$, and thereby we get

$$
f_{1}(\lambda) \leq f_{1}\left(\lambda_{1}\right) \quad \text { for } \lambda_{1} \in(1,+\infty) \text {. }
$$

On the other hand, we deduce from $f_{2}\left(\lambda_{1}\right)=0$ that $\lambda_{1}^{p / 2-q / 2}=q^{2} / p^{2}$, thus, we have

$$
\begin{aligned}
f_{1}\left(\lambda_{1}\right) & =2 p^{2} \lambda_{1}^{p / 2-q / 2} \lambda_{1}^{q}-q(p+q) \lambda_{1}^{q}+q(p-q) \\
& =2 p^{2} \cdot \frac{q^{2}}{p^{2}} \cdot \lambda_{1}^{q}-q(p+q) \lambda_{1}^{q}+q(p-q) \\
& =q(p-q)\left(1-\lambda_{1}^{q}\right)<0
\end{aligned}
$$

which implies $f_{1}(\lambda)<0$, i.e., $g_{1}^{\prime}(\lambda)<0$ for $\lambda \in[1,+\infty)$.

We conclude that $g_{1}(\lambda)$ is decreasing on $[1,+\infty)$. It, therefore, follows that $g_{1}(\lambda) \leq$ $g_{1}(1) \leq 0$.

Case 2. $p q=0$.

It is easy to verify that:

If $p>q=0$ (it implies that $p+q>0$ ), then $g_{1}(\lambda)=p\left(\lambda^{p}-1\right) \geq 0$.

If $0=p>q$ (it implies that $p+q<0$ ), then $g_{1}(\lambda)=q \lambda^{-q / 2}\left(1-\lambda^{q}\right) \leq 0$.

If $0=p=q$ (it implies that $p+q=0$ ), then $g_{1}(\lambda)=0$.

The proof of Lemma 3 is complete.

Lemma 4 Let $p, q \in \mathbb{R}, p>q, \lambda \geq 1$, and let

$$
g_{2}(\lambda)=(p-q) \lambda^{p+q}+(p+q) \lambda^{p}-(p+q) \lambda^{q}-(p-q) .
$$

Then $g_{2}(\lambda) \geq 0$ for $p+q \geq 0$, and $g_{2}(\lambda) \leq 0$ for $p+q \leq 0$.

Proof Let $h(\lambda)=\lambda^{1-q} g_{2}^{\prime}(\lambda)$. It follows from a simple computation that

$$
\begin{aligned}
& h(\lambda)=(p-q)(p+q) \lambda^{p}+p(p+q) \lambda^{p-q}-q(p+q), \\
& h(1)=2(p-q)(p+q), \\
& h^{\prime}(\lambda)=p(p-q)(p+q) \lambda^{p-1}\left(1+\lambda^{-q}\right) .
\end{aligned}
$$

Case 1. $p q \neq 0$.

(1) If $p+q=0$, then

$$
g_{2}(\lambda)=(p+p) \lambda^{p-p}+(p-p) \lambda^{p}-(p-p) \lambda^{-p}-(p+p)=0 .
$$

(2) If $p+q>0$, then from the hypothesis $p>q$ we find that $p>(p+q) / 2>0$, and then we derive from the expressions $h^{\prime}(\lambda), h(1)$ that, for $\lambda \geq 1$,

$$
h^{\prime}(\lambda)>0, \quad h(1)>0,
$$

which shows that $h(\lambda)$ is increasing on $[1,+\infty)$, so, we have $h(\lambda) \geq h(1)>0$ for $\lambda \geq 1$. 
Thus, we obtain $g_{2}^{\prime}(\lambda)>0$ for $\lambda \geq 1$. Now, from the fact that $g_{2}(\lambda)$ is increasing on $[1,+\infty)$, we obtain $g_{2}(\lambda) \geq g_{2}(1)=0(\lambda \geq 1)$.

(3) If $p+q<0$ and $p>0$, then

$$
h^{\prime}(\lambda) \leq 0, \quad h(1)<0 .
$$

Note that $h(\lambda)$ is decreasing on $[1,+\infty)$, we get $h(\lambda) \leq h(1)<0$ for $\lambda \geq 1$, and then we get $g_{2}^{\prime}(\lambda)<0$ for $\lambda \geq 1$.

Finally, in view of the fact that $g_{2}(\lambda)$ is decreasing on $[1,+\infty)$, we deduce $g_{2}(\lambda) \leq g_{2}(1)=0$ $(\lambda \geq 1)$.

(4) If $p+q<0$ and $p<0$, then

$$
h^{\prime}(\lambda)>0, \quad h(1)<0, \quad \lim _{\lambda \rightarrow+\infty} \frac{h(\lambda)}{\lambda^{p-q}}=p(p+q)>0 .
$$

By the monotonicity and the continuity of $h(\lambda)$, we find that there exists $\lambda_{2} \in(1,+\infty)$ such that $h\left(\lambda_{2}\right)=0$, which implies that $h(\lambda)<0$ for $\lambda \in\left[1, \lambda_{2}\right)$, and $h(\lambda)>0$ for $\lambda \in\left(\lambda_{2},+\infty\right)$.

We hereby deduce that $g_{2}^{\prime}(\lambda)<0$ for $\lambda \in\left[1, \lambda_{2}\right)$, and $g_{2}^{\prime}(\lambda)>0$ for $\lambda \in\left(\lambda_{2},+\infty\right)$. Further, we conclude that $g_{2}(\lambda)$ is decreasing on $\left[1, \lambda_{2}\right)$ and increasing on $\left(\lambda_{2},+\infty\right)$.

Therefore, we obtain

$$
g_{2}(\lambda) \leq \max \left\{g_{2}(1), \lim _{\lambda \rightarrow+\infty} g_{2}(\lambda)\right\}=\max \{0,-(p-q)\}=0
$$

Case 2. $p q=0$.

It is easy to verify that:

If $p>q=0$ (it implies that $p+q>0$ ), then $g_{2}(\lambda)=2 p\left(\lambda^{p}-1\right) \geq 0$.

If $0=p>q$ (it implies that $p+q<0$ ), then $g_{2}(\lambda)=2 q\left(1-\lambda^{q}\right) \leq 0$.

If $0=p=q$ (it implies that $p+q=0$ ), then $g_{2}(\lambda)=0$.

This completes the proof of Lemma 4.

\section{Main result}

The main result of this paper is given by Theorem 1 below.

Theorem 1 For fixed $(p, q, w) \in \mathbb{R}^{3}$,

(1) if $p+q \geq 0$ and $w \geq 0$, then the generalized Gini-Heronian means $H_{p, q, w}(x, y)$ are Schur-geometric convex for $(x, y) \in \mathbb{R}_{++}^{2}$;

(2) if $p+q \leq 0$ and $w \geq 0$, then the generalized Gini-Heronian means $H_{p, q, w}(x, y)$ are Schur-geometric concave for $(x, y) \in \mathbb{R}_{++}^{2}$.

Proof We consider the following two cases.

Case 1. If $p=q$, then

$$
H_{p, q, w}(x, y)=\exp \left(\frac{x^{p} \ln x+(w / 2)(x y)^{p / 2} \ln (x y)+y^{p} \ln y}{x^{p}+w(x y)^{p / 2}+y^{p}}\right) .
$$

Differentiating $H_{p, q, w}(x, y)$ with respect to $x$ and $y$, respectively, we obtain

$$
\frac{\partial H}{\partial x}=\frac{H_{p, q, w}(x, y) G_{1}(x, y, p, w)}{x\left[x^{p}+w(x y)^{p / 2}+y^{p}\right]^{2}}, \quad \frac{\partial H}{\partial y}=\frac{H_{p, q, w}(x, y) G_{2}(x, y, p, w)}{y\left[x^{p}+w(x y)^{p / 2}+y^{p}\right]^{2}},
$$


where

$$
\begin{aligned}
G_{1}(x, y, p, w)= & {\left[p x^{p} \ln x+x^{p}+(p w / 4)(x y)^{p / 2} \ln (x y)+(w / 2)(x y)^{p / 2}\right]\left[x^{p}+w(x y)^{p / 2}+y^{p}\right] } \\
& -p\left[x^{p} \ln x+(w / 2)(x y)^{p / 2} \ln (x y)+y^{p} \ln y\right]\left[x^{p}+(w / 2)(x y)^{p / 2}\right], \\
G_{2}(x, y, p, w)= & {\left[p y^{p} \ln y+y^{p}+(p w / 4)(x y)^{p / 2} \ln (x y)+(w / 2)(x y)^{p / 2}\right]\left[x^{p}+w(x y)^{p / 2}+y^{p}\right] } \\
& -p\left[x^{p} \ln x+(w / 2)(x y)^{p / 2} \ln (x y)+y^{p} \ln y\right]\left[y^{p}+(w / 2)(x y)^{p / 2}\right] .
\end{aligned}
$$

By calculation, it follows that

$$
\Lambda:=(x-y)\left(x \frac{\partial H}{\partial x}-y \frac{\partial H}{\partial y}\right)=\frac{(x-y) H_{p, q, w}(x, y) F(x, y, p, w)}{\left[x^{p}+w(x y)^{p / 2}+y^{p}\right]^{2}},
$$

where

$$
\begin{aligned}
F(x, y, p, w)= & G_{1}(x, y, p, w)-G_{2}(x, y, p, w) \\
= & \left(p x^{p} \ln x+x^{p}-p y^{p} \ln y-y^{p}\right)\left[x^{p}+w(x y)^{p / 2}+y^{p}\right] \\
& -p\left[x^{p} \ln x+(w / 2)(x y)^{p / 2} \ln (x y)+y^{p} \ln y\right]\left(x^{p}-y^{p}\right) \\
= & (x y)^{p / 2}\left[x^{p}-y^{p}+(p / 2)\left(x^{p}+y^{p}\right) \ln (x / y)\right] w+2 p x^{p} y^{p} \ln (x / y)+x^{2 p}-y^{2 p} .
\end{aligned}
$$

Note that the expression $\Lambda$ is symmetric in $x$ and $y$, without loss of generality we assume that $x \geq y$.

Setting $\lambda=x / y, \lambda \geq 1$, we have

$$
F(x, y, p, w)=(x y)^{p / 2} y^{p}\left[\lambda^{p}-1+(p / 2)\left(\lambda^{p}+1\right) \ln \lambda\right] w+y^{2 p}\left(2 p \lambda^{p} \ln \lambda+\lambda^{2 p}-1\right) .
$$

In addition, it is easy to show that

$$
\begin{array}{ll}
{\left[\lambda^{p}-1+(p / 2)\left(\lambda^{p}+1\right) \ln \lambda\right] w+y^{2 p}\left(2 p \lambda^{p} \ln \lambda+\lambda^{2 p}-1\right) \geq 0} & \text { for } p \geq 0 \text { and } w \geq 0, \\
{\left[\lambda^{p}-1+(p / 2)\left(\lambda^{p}+1\right) \ln \lambda\right] w+y^{2 p}\left(2 p \lambda^{p} \ln \lambda+\lambda^{2 p}-1\right) \leq 0} & \text { for } p \leq 0 \text { and } w \geq 0 .
\end{array}
$$

This yields

$$
\Lambda \geq 0 \text { for } p \geq 0, w \geq 0 \text { and } \Lambda \leq 0 \text { for } p \leq 0, w \geq 0 .
$$

Case 2. If $p \neq q$, then

$$
H_{p, q, w}(x, y)=\left(\frac{x^{p}+w(x y)^{p / 2}+y^{p}}{x^{q}+w(x y)^{q / 2}+y^{q}}\right)^{1 /(p-q)} .
$$

Differentiating $H_{p, q, w}(x, y)$ with respect to $x$ and $y$, respectively, we get

$$
\begin{aligned}
& \frac{\partial H}{\partial x}=\frac{H_{p, q, w}(x, y)}{p-q}\left[\frac{p x^{p-1}+(p w / 2) y(x y)^{(p / 2)-1}}{x^{p}+w(x y)^{p / 2}+y^{p}}-\frac{q x^{q-1}+(q w / 2) y(x y)^{(q / 2)-1}}{x^{q}+w(x y)^{q / 2}+y^{q}}\right], \\
& \frac{\partial H}{\partial y}=\frac{H_{p, q, w}(x, y)}{p-q}\left[\frac{p y^{p-1}+(p w / 2) x(x y)^{(p / 2)-1}}{x^{p}+w(x y)^{p / 2}+y^{p}}-\frac{q y^{q-1}+(q w / 2) x(x y)^{(q / 2)-1}}{x^{q}+w(x y)^{q / 2}+y^{q}}\right] .
\end{aligned}
$$


Direct calculation gives

$$
\begin{aligned}
\Lambda & :=(x-y)\left(x \frac{\partial H}{\partial x}-y \frac{\partial H}{\partial y}\right) \\
& =\frac{H_{p, q, w}(x, y)(x-y)}{p-q}\left[\frac{p\left(x^{p}-y^{p}\right)}{x^{p}+w(x y)^{p / 2}+y^{p}}-\frac{q\left(x^{q}-y^{q}\right)}{x^{q}+w(x y)^{q / 2}+y^{q}}\right] .
\end{aligned}
$$

It is obvious that the expression $\Lambda$ is symmetric with respect to $p$ and $q$ (it is also symmetric with respect to $x$ and $y$ ), and without loss of generality we assume that $x \geq y$ and $p>q$ in the following discussion.

Simplifying the expression $\Lambda$, we obtain

$$
\Lambda=\frac{H_{p, q, w}(x, y)(x-y) F(x, y, p, q, w)}{(p-q)\left(x^{p}+w(x y)^{p / 2}+y^{p}\right)\left(x^{q}+w(x y)^{q / 2}+y^{q}\right)},
$$

where

$$
\begin{aligned}
F(x, y, p, q, w)= & p\left(x^{p}-y^{p}\right)\left(x^{q}+w(x y)^{q / 2}+y^{q}\right)-q\left(x^{q}-y^{q}\right)\left(x^{p}+w(x y)^{p / 2}+y^{p}\right) \\
= & \left(p x^{p+(q / 2)} y^{q / 2}-q x^{q+(p / 2)} y^{p / 2}+q x^{p / 2} y^{q+(p / 2)}-p x^{q / 2} y^{p+(q / 2)}\right) w \\
& +(p-q) x^{p+q}+(p+q) x^{p} y^{q}-(p+q) x^{q} y^{p}-(p-q) y^{p+q} \\
= & y^{p+q}\left[p(x / y)^{p+(q / 2)}-q(x / y)^{q+(p / 2)}+q(x / y)^{p / 2}-p(x / y)^{q / 2}\right] w \\
& +y^{p+q}\left[(p-q)(x / y)^{p+q}+(p+q)(x / y)^{p}-(p+q)(x / y)^{q}-(p-q)\right] .
\end{aligned}
$$

Setting $\lambda=x / y, \lambda \geq 1$, then

$$
F(x, y, p, q, w)=y^{p+q}\left[\lambda^{q / 2} g_{1}(\lambda) w+g_{2}(\lambda)\right]
$$

where

$$
\begin{aligned}
& g_{1}(\lambda)=p \lambda^{p}-q \lambda^{p / 2+q / 2}+q \lambda^{p / 2-q / 2}-p, \\
& g_{2}(\lambda)=(p-q) \lambda^{p+q}+(p+q) \lambda^{p}-(p+q) \lambda^{q}-(p-q) .
\end{aligned}
$$

It follows from Lemmas 3 and 4 that

$$
\begin{array}{ll}
F(x, y, p, q, w) \geq 0 & \text { for } p+q \geq 0 \text { and } w \geq 0 \\
F(x, y, p, q, w) \leq 0 & \text { for } p+q \leq 0 \text { and } w \geq 0 .
\end{array}
$$

This evidently implies that

$$
\Lambda \geq 0 \text { for } p+q \geq 0, w \geq 0 \text { and } \quad \Lambda \leq 0 \text { for } p+q \leq 0, w \geq 0 .
$$

By using the conclusions obtained in Cases 1 and 2 together with an application of Lemma 1, we are led to the desired results:

$H_{p, q, w}(x, y)$ is Schur-geometric convex on $\mathbb{R}_{++}^{2}$ when $p+q \geq 0$ and $w \geq 0$. Furthermore, $H_{p, q, w}(x, y)$ is Schur-geometric concave on $\mathbb{R}_{++}^{2}$ when $p+q \leq 0$ and $w \geq 0$. 
The proof of Theorem 1 is thus completed.

Remark 1 The main result of Theorem E would follow as a special case of Theorem 1 $(q=0)$. Namely, the result stated in Theorem 1 is an extension of the result given in [7].

\section{An application}

As an application of Theorem 1, we establish the following interesting inequalities for generalized Gini-Heronian means.

Theorem 2 Let $0<x \leq y$, and let $1 / 2 \leq t_{2} \leq t_{1} \leq 1$ or $0 \leq t_{1} \leq t_{2} \leq 1 / 2$.

$$
\begin{aligned}
& \text { If } p+q \geq 0 \text { and } w \geq 0 \text {, then } \\
& \begin{aligned}
H_{p, q, w}(\sqrt{x y}, \sqrt{x y}) & \leq H_{p, q, w}\left(y^{t_{2}} x^{1-t_{2}}, x^{t_{2}} y^{1-t_{2}}\right) \\
& \leq H_{p, q, w}\left(y^{t_{1}} x^{1-t_{1}}, x^{t_{1}} y^{1-t_{1}}\right) \leq H_{p, q, w}(x, y) .
\end{aligned}
\end{aligned}
$$

If $p+q \leq 0$ and $w \geq 0$, then

$$
\begin{aligned}
H_{p, q, w}(\sqrt{x y}, \sqrt{x y}) & \geq H_{p, q, w}\left(y^{t_{2}} x^{1-t_{2}}, x^{t_{2}} y^{1-t_{2}}\right) \\
& \geq H_{p, q, w}\left(y^{t_{1}} x^{1-t_{1}}, x^{t_{1}} y^{1-t_{1}}\right) \geq H_{p, q, w}(x, y),
\end{aligned}
$$

where $H_{p, q, w}(x, y)$ is given by

$$
H_{p, q, w}(x, y)= \begin{cases}\left(\frac{\left.x^{p}+w(x y)\right)^{p / 2}+y^{p}}{\left.x^{q}+w(x)\right)^{q / 2}+y^{q}}\right)^{1 /(p-q)}, & p \neq q, \\ \exp \left(\frac{x^{p} \ln x+(w / 2)(x y)^{p / 2} \ln (x y)+y^{p} \ln y}{x^{p}+w(x y)^{p / 2}+y^{p}}\right), & p=q .\end{cases}
$$

Proof Using Lemma 2 with a substitution $a=\ln x, b=\ln y$ gives

$$
\begin{aligned}
\left(\frac{\ln x+\ln y}{2}, \frac{\ln x+\ln y}{2}\right) & \prec\left(t_{2} \ln y+\left(1-t_{2}\right) \ln x, t_{2} \ln x+\left(1-t_{2}\right) \ln y\right) \\
& \prec\left(t_{1} \ln y+\left(1-t_{1}\right) \ln x, t_{1} \ln x+\left(1-t_{1}\right) \ln y\right) \prec(\ln x, \ln y),
\end{aligned}
$$

which is equivalent to

$$
\begin{aligned}
(\ln \sqrt{x y}, \ln \sqrt{x y}) & \prec\left(\ln \left(y^{t_{2}} x^{1-t_{2}}\right), \ln \left(x^{t_{2}} y^{1-t_{2}}\right)\right) \\
& \prec\left(\ln \left(y^{t_{1}} x^{1-t_{1}}\right), \ln \left(x^{t_{1}} y^{1-t_{1}}\right)\right) \prec(\ln x, \ln y) .
\end{aligned}
$$

On the other hand, we derive from Theorem 1 that

$H_{p, q, w}(x, y)$ is Schur-geometric convex for $p+q \geq 0$ and $w \geq 0, H_{p, q, w}(x, y)$ is

Schur-geometric concave for $p+q \leq 0$ and $w \geq 0$.

Thus, from Definition 2, it follows that

$$
\begin{aligned}
H_{p, q, w}(\sqrt{x y}, \sqrt{x y}) & \leq H_{p, q, w}\left(y^{t_{2}} x^{1-t_{2}}, x^{t_{2}} y^{1-t_{2}}\right) \\
& \leq H_{p, q, w}\left(y^{t_{1}} x^{1-t_{1}}, x^{t_{1}} y^{1-t_{1}}\right) \leq H_{p, q, w}(x, y)
\end{aligned}
$$


for $p+q \geq 0$ and $w \geq 0$; and that

$$
\begin{aligned}
H_{p, q, w}(\sqrt{x y}, \sqrt{x y}) & \geq H_{p, q, w}\left(y^{t_{2}} x^{1-t_{2}}, x^{t_{2}} y^{1-t_{2}}\right) \\
& \geq H_{p, q, w}\left(y^{t_{1}} x^{1-t_{1}}, x^{t_{1}} y^{1-t_{1}}\right) \geq H_{p, q, w}(x, y)
\end{aligned}
$$

for $p+q \leq 0$ and $w \geq 0$.

The above-mentioned inequalities are the required inequalities in Theorem 2. This completes the proof of Theorem 2 .

Putting $q=0$ in Theorem 2 gives the following inequalities.

Theorem 3 Let $0<x \leq y, w \geq 0$, and let $1 / 2 \leq t_{2} \leq t_{1} \leq 1$ or $0 \leq t_{1} \leq t_{2} \leq 1 / 2$. Then, for $p \geq 0$, we have the inequality

$$
\begin{aligned}
G(x, y) & \leq H_{p, w}\left(y^{t_{2}} x^{1-t_{2}}, x^{t_{2}} y^{1-t_{2}}\right) \\
& \leq H_{p, w}\left(y^{t_{1}} x^{1-t_{1}}, x^{t_{1}} y^{1-t_{1}}\right) \leq H_{p, w}(x, y) .
\end{aligned}
$$

Furthermore, for $p \leq 0$ we have the inequality

$$
\begin{aligned}
G(x, y) & \geq H_{p, w}\left(y^{t_{2}} x^{1-t_{2}}, x^{t_{2}} y^{1-t_{2}}\right) \\
& \geq H_{p, w}\left(y^{t_{1}} x^{1-t_{1}}, x^{t_{1}} y^{1-t_{1}}\right) \geq H_{p, w}(x, y),
\end{aligned}
$$

where $G(x, y)=\sqrt{x y}, H_{p, w}(x, y)$ is given by

$$
H_{p, w}(x, y)= \begin{cases}\left(\frac{x^{p}+w(x y)^{p / 2}+y^{p}}{w+2}\right)^{1 / p}, & p \neq 0, \\ \sqrt{x y}, & p=0 .\end{cases}
$$

Remark 2 Inequalities (4.3) and (4.4) were first presented by Shi et al. in [7]. It is obvious that the inequalities given in Theorem 2 provide the generalized versions of these inequalities.

\section{Competing interests}

The authors declare that they have no conflicts of interest to this work.

\section{Authors' contributions}

All authors contributed equally to the writing of this paper. All authors read and approved the final manuscript.

\section{Author details}

${ }^{1}$ Department of Mathematics and Computer Science, Longyan University, Longyan, 364012, P.R. China. ${ }^{2}$ School of Mathematics and Computation Science, Hunan City University, Yiyang, 413000, P.R. China. ${ }^{3}$ Department of Mathematics, University of Texas-Pan American, Edinburg, TX 78539, USA. ${ }^{4}$ Gangtou Middle School, Fuqing, 350317, P.R. China.

\section{Acknowledgements}

This research was supported by the Natural Science Foundation of China under Grants 11171307 and 61374086, the Natural Science Foundation of Zhejiang Province under Grant LY13A010004 and the Foundation of Scientific Research Project of Fujian Province Education Department under Grants JK2013051 and JK2012049. 


\section{References}

1. Marshall, AW, Olkin, I: Inequalities: Theory of Majorization and Its Applications. Academic Press, New York (1979)

2. Shi, H-N, Zhang, J: Some new judgement theorems of Schur geometric and Schur harmonic convexities for a class of symmetric functions. J. Inequal. Appl. 2013, Article ID 527 (2013)

3. Shi, H-N, Zhang, J: Schur-convexity of dual form of some symmetric functions. J. Inequal. Appl. 2013, Article ID 295 (2013)

4. Shi, H-N, Zhang, J: Schur-convexity, Schur geometric and Schur harmonic convexities of dual form of a class symmetric functions. J. Math. Inequal. 8(2), 349-358 (2014)

5. Zhang, XM, Yang, ZH: Differential criterion of $n$-dimensional geometrically convex functions. J. Appl. Anal. 13(2), 197-208 (2007)

6. Chu, Y, Zhang, X-M, Wang, GD: The Schur geometrical convexity of the extended mean values. J. Convex Anal. 15(4), 707-718 (2008)

7. Shi, H-N, Bencze, M, Wu, S-H, Li, D-M: Schur convexity of generalized Heronian means involving two parameters. J. Inequal. Appl. 2008, Article ID 879273 (2008)

8. Gini, C: Diuna formula comprensiva delle medie. Metron 13, 3-22 (1938)

9. Sándor, J: The Schur-convexity of Stolarsky and Gini means. Banach J. Math. Anal. 1(2), 212-215 (2007)

10. Wang, Z-H: The sufficient and necessary condition of the Schur-convexity and Schur-geometric-convexity of the Gini means. J. Beijing Inst. Educ. 2(5), 1-3 (2007)

11. Shi, H-N, Jiang, Y-M, Jiang, W-D: Schur-convexity and Schur-geometrically concavity of Gini means. Comput. Math. Appl. 57(2), 266-274 (2009)

12. Chu, Y-M, Xia, W-F: Solution of an open problem for Schur convexity or concavity of the Gini mean values. Sci. China Math. 52(10), 2099-2106 (2009)

13. Xia, W-F, Chu, Y-M: The Schur convexity of Gini mean values in the sense of harmonic mean. Acta Math. Sci., Ser. B 31(3), 1103-1112(2011)

14. Yang, Z-H: Schur power convexity of Gini means. Bull. Korean Math. Soc. 50(2), 485-498 (2013)

15. Alzer, $H$, Janous, W: Solution of problem 8. Crux Math. 13, 173-178 (1987)

16. Mao, Q-J: Dual means, logarithmic and Heronian dual means of two positive numbers. J. Suzhou Coll. Educ. 16, 82-85 (1999)

17. Janous, W: A note on generalized Heronian means. Math. Inequal. Appl. 4(3), 369-375 (2001)

18. Jia, G, Cao, J: A new upper bound of the logarithmic means. J. Inequal. Pure Appl. Math. 4(4), Article ID 80 (2003)

19. Li, D-M, Gu, Ch, Shi, H-N: Schur convexity of the power-type generalization of Heronian means. Math. Pract. Theory 36(9), 387-390 (2006)

20. Fu, L-L, Xi, B-Y, Srivastava, HM: Schur-convexity of the generalized Heronian means involving two positive numbers. Taiwan. J. Math. 15(6), 2721-2731 (2011)

21. Yang, Z-H: Schur power convexity of the Daróczy means. Math. Inequal. Appl. 16(3), 751-762 (2013)

22. Mortici, C: Arithmetic mean of values and value at mean of arguments for convex functions. ANZIAM J. 50(1), 137-141 (2008)

23. Zhang, X-M: Geometrically Convex Functions. Anhui University Press, Hefei (2004)

10.1186/1029-242X-2014-413

Cite this article as: Deng et al.: Schur-geometric convexity of the generalized Gini-Heronian means involving three parameters. Journal of Inequalities and Applications 2014, 2014:413

\section{Submit your manuscript to a SpringerOpen ${ }^{\circ}$ journal and benefit from:}

- Convenient online submission

- Rigorous peer review

- Immediate publication on acceptance

- Open access: articles freely available online

- High visibility within the field

- Retaining the copyright to your article 